\title{
Role of DDR2 ECD Oligomerization in Binding to Collagen
}

Carolyn Wang ${ }^{1}$, David Yeung ${ }^{1}$, Jack Wellerming ${ }^{2}$, Andrew Herr ${ }^{3}$, Jeanette Miller ${ }^{3}$, Rafael Fridman ${ }^{4}$ and Gunjan Agarwal ${ }^{1,2}$

1. Biomedical Engineering Department, the Ohio State University Columbus, Ohio 43210, USA

2. Biophysics Program, the Ohio State University, Columbus, Ohio 43210, USA

3. Department of Biochemistry and Molecular Genetics, University of Cincinnati, Cincinnati, OH, USA

4. Department of Pathology, Wayne State University, Detroit, MI, USA

Discoidin Domain Receptors (DDR1 and DDR2) are widely expressed receptor tyrosine kinases (RTK) that regulate cell differentiation, proliferation and migration and remodeling of the extracellular matrix [1]. Collagen(s) are the only known ligand for DDRs [2]. Both the collagen binding domains of the receptors (Figure 1) as well as their binding site on the collagen triple helix have been elucidated in recent years [3]. However, the role of receptor oligomerization in DDR-collagen interaction is not completely understood. This is especially important as receptor oligomerization can modulate receptorligand binding as well as receptor phosphorylation and activation and downstream-signaling events.

Previous studies by us [4] and others [5] have shown that (i) dimerization of DDR1 is crucial for its binding to collagen, (ii) DDR1 exists as a dimer on the cell surface, and (iii) DDR1 undergoes further oligomerization upon collagen binding. Further, we recently elucidated how the oligomerization of DDR1 is mediated by its extra-cellular domain (ECD) [6]. In this study we examined the role of DDR2 ECD oligomerization in its binding to monomeric triple-helical collagen type 1.

Recombinant proteins expressing DDR2 ECD as a monomer (DDR2-V5-His) or Fc-tagged dimers (DDR2-Fc) were expressed and purified from mammalian cells. Antibody (anti-Fc) induced oligomers of DDR2-Fc were also used. Solid phase binding assays showed that binding of recombinant DDR2ECD to immobilized collagen type 1 increased with its oligomeric state. For instance, oligomers of recombinant DDR2-Fc bound more strongly to collagen compared to dimeric DDR1-Fc or DDR2-V5 alone. As another manifestation of binding, oligomeric DDR2 inhibited fibrillogenesis of collagen more strongly than dimeric or monomeric forms. To examine how the differences in the oligomeric state of DDR2 impacts its binding to collagen, we carried out single molecule atomic-force microscopy (AFM) experiments. The size of DDR2-ECD particles bound to collagen was evaluated by measuring their topographic heights from section profiles. As a control, recombinant DDR2-ECD proteins were also imaged and analyzed before binding to collagen. Our AFM results revealed that DDR2 ECD did not undergo further oligomerization upon binding to collagen (Figure 2). This feature was consistently observed for monomeric, dimeric as well as oligomeric DDR2 ECD.

Our results provide novel insights in the interaction of DDR1 vs. DDR2 with collagen. Both receptors demonstrate a higher affinity in binding to collagen when their ECD is in a higher oligomeric state. However, in sharp contrast to DDR1 ECD which underwent further oligomerization upon binding to collagen, DDR2 ECD did not. It remains to be examined if either the juxta- or intracellular domains of DDR2 mediate receptor oligomerization upon ligand binding. 


\section{References:}

[1] H. L. Fu et al, "Discoidin domain receptors: unique receptor tyrosine kinases in collagen-mediated signaling”, J. Biol. Chem 288 (2013), no. 11, p. 7430-7.

[2] W.Vogel, et al, "The Discoidin Domain Receptor Tyrosine Kinases Are Activated by Collagen", Mol. Cell 1 (1997), no.1, p. 13-23.

[3] H. Xu, et al, "Collagen binding specificity of the discoidin domain receptors: binding sites on collagens II and III and molecular determinants for collagen IV recognition by DDR1", Matrix Biol 30 (2011), no. 1, p. 16-26.

[4] C. Mihai, "Mapping of DDR1 Distribution and Oligomerization on the Cell Surface by FRET Micrscopy”, J. Miol. Biol. 30 (2009), no. 2, p. 432-435.

[5] N.A. Noordeen, et al, "A transmembrane leucine zipper is required for activation of the dimeric receptor tyrosine kinase DDR1”, J. Biol. Chem 281 (2006), no. 32, p. 22744-51.

[6] D. Yeung, et al, "Oligomerization of DDR1 ECD affects receptor-ligand binding”, J. Struct. Biol (2013), p. 1-6.

[7] The authors acknowledge funding from NSF CMMI award 1201111.

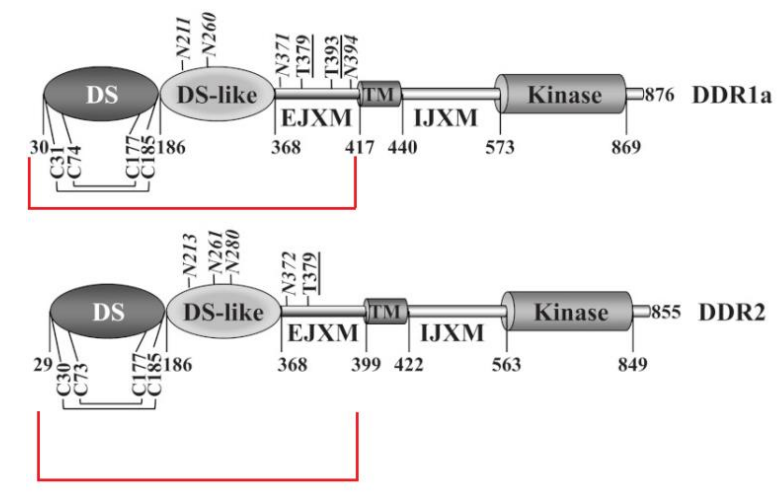

Figure 1. Structure of DDR1a and DDR2. DDRs contain a cytosolic, transmembrane, and extracellular domain (ECD) with a juxta-membrane region that is unusually long for RTKs. The recombinant DDRs used in our study contained only the ECD (red brackets).
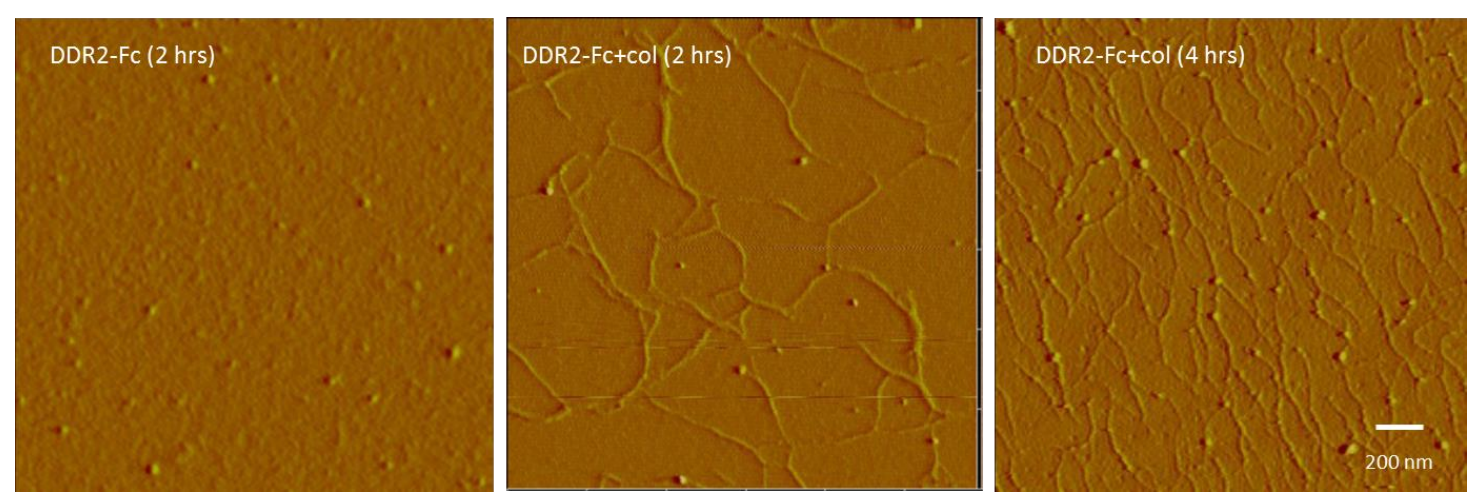

Figure 2. AFM images of recombinant DDR2-Fc with and without incubation with monomeric collagen type 1 at $4^{\circ} \mathrm{C}$ for durations as indicated. The size of the DDR2-Fc proteins remains unchanged before and after binding to collagen. 